\title{
Aligning linac accelerating structures using a copropagating octupolar mode
}

\author{
J. Ögren ${ }^{*}$ and V. Ziemann \\ Department of Physics and Astronomy, Uppsala University, Box 516, 75120 Uppsala, Sweden
}

(Received 22 June 2016; published 16 October 2017)

\begin{abstract}
We propose a novel method to align accelerating structures such as those used in the Compact Linear Collider (CLIC) by exploiting a mode that copropagates with the normal accelerating mode. This mode has an octupolar dependence in the transverse direction and is caused by radial waveguides intended to damp higher-order modes. The nonlinear dependence of the octupolar mode makes it possible to determine the center of the structure from the nonlinear dependence of the transverse kick, observed on a downstream beam position monitor, while changing the transverse position of the beam with respect to the accelerating structures. We discuss the method, its tolerances and disentangling the individual misalignments of two adjacent accelerating structures that are powered from a single source.
\end{abstract}

DOI: 10.1103/PhysRevAccelBeams.20.102801

\section{INTRODUCTION}

The Compact Linear Collider (CLIC) [1] is a candidate for a future lepton collider to probe physics on the $\mathrm{TeV}$ scale beyond LHC. In order to achieve the high energies in a reasonable length, normal-conducting accelerating structures with accelerating gradients of $100 \mathrm{MV} / \mathrm{m}$ are required and have been experimentally shown [2] to have adequate reliability. The fact that the structures are normalconducting implies that the accelerator is operated with macropulse length below $1 \mu \mathrm{s}$ and at a repetition rate of $50 \mathrm{~Hz}$. In order to achieve luminosities above $10^{34} / \mathrm{cm}^{2} \mathrm{~s}$ the beam size at the interaction point must be squeezed to nanometer sizes. The beam size depends to a large extent on the beam emittance that is initially determined by the damping rings. Consequently, the emittance must be preserved while the beam travels down the linear accelerator, but is, on the other hand, adversely affected by transversely misaligned quadrupoles and acceleration structures. They cause transverse wakefields that deflect the beam and ultimately lead to an increased emittance and reduced luminosity. Therefore the acceleration structures must be aligned with utmost precision and the topic has been extensively studied theoretically [3-5] and experimentally [6-8]. Even the intrinsic mechanical alignment [9] was addressed. For CLIC there is ongoing research for using wakefield monitors for beam alignment [10].

In this report we discuss a novel method to complement other alignment methods for the accelerating structures. It is based on the existence of a mode in the accelerating

\footnotetext{
*Corresponding author. jim.ogren@physics.uu.se

Published by the American Physical Society under the terms of the Creative Commons Attribution 3.0 License. Further distribution of this work must maintain attribution to the author $(s)$ and the published article's title, journal citation, and DOI.
}

structures that is copropagating with the accelerating mode but shifted 90 degrees in phase and transversely has an octupolar amplitude dependence. The existence of the octupolar mode is known, both from theoretical investigations [11] and experimentally [12,13]. In [13] we thoroughly analyzed the effect of the octupolar mode on the beam and devised a method to extract information about the beam matrix by scanning the beam across the entrance face of the structure. Moreover, we determined the strength of the octupolar mode. The fact that the field is nonlinear leads to a nonlinear deflection of the beam and can be used to determine the center of the accelerating cells, which for the accelerating structure coincides with the center of the irises with micrometer tolerance. The method is somewhat inspired by using the nonlinear beam-beam deflections to center the counterpropagating beams at the interaction point of the SLC $[14,15]$.

We propose to scan the beam transversely inside the accelerating structures while measuring the beam position downstream. From the beam position shifts, i.e., difference in beam position for deflected and nondeflected beam, we can determine the electromagnetic center in the structure. In CLIC the radio-frequency (rf) power is generated by deceleration of a drive beam in 24 sectors each about $900 \mathrm{~m}$ long. In the power extraction structures there is a mechanism that allows rf to be switched on and off [16], which means beam position measurements for deflected and nondeflected beam can be made shot-to-shot. This eliminates many systematic errors and lowers the sensitivity to beam jitter. For this analysis we will use the CLIC main beam parameters from Table I. We point out that considering the large number of accelerating structures in CLIC ( 140, 000 in the $3 \mathrm{TeV}$ version) method that does not require extra hardware is very attractive. Furthermore, the method is easy to automate.

The magnitude of the kick due to the octupolar component scales inversely with the beam energy and would 
TABLE I. CLIC beam parameters at the beginning of the main linac [1].

\begin{tabular}{lr}
\hline \hline Parameter [unit] & value \\
\hline Beam energy [GeV] & 9 \\
Beta function [m] & 10 \\
Normalized emittance, horizontal [nm] & 600 \\
Normalized emittance, vertical [nm] & 10 \\
\hline \hline
\end{tabular}

render the method impractical at high energy. This problem, however, can be circumvented by powering only one of the 24 drive beam sectors at a time and running the entire linac at or close to the injection energy of $9 \mathrm{GeV}$. Moreover, within the sector under consideration all power extraction structures except the tested should be turned off. Of course this requires suitably scaling the static magnetic elements such as quadrupoles. Assuming that the magnetic centers of the magnetic elements are unaffected by this scaling the alignment of the acceleration structures then works for the entire linac. This procedure is invasive, but only needs to done once at the start of operation to ensure proper alignment. We investigated stable operation of the entire CLIC linac close to injection energy with simulations using placet [17] and found that a beam with an injection error of about $10 \mu \mathrm{m}$ in both planes experiences only a moderate growth during one sector. Moreover, operating the already aligned sectors with $10 \%$ of the nominal gradient and 80 degree off-crest leads to a moderate beam size increase to reach the next sector to be aligned. Running the linac at low energy will constitute an operational challenge and will likely be difficult. Aligning one sector at a time, before progressing to the next, in order to prevent excessive wakefield tails and emittance growth, will certainly be necessary. We expect to empirically optimize parameters in the upstream sectors to balance the requirement for stable operation at low beam energy with the requirement for adequate resolution for our alignment method.

In the following section we elaborate on the theory and then apply it to alignment of the accelerating structures. We first show the method for a single structure [18] and then we expand the error analysis and apply the method to two consecutive structures.

\section{TRANSVERSE DEFLECTIONS}

The accelerating structures, such as those used in CLIC, support an octupole component of the rf fields that is copropagating with the beam $[11,12]$. Therefore, the effect on the beam can equivalently be described by a kick from a static magnetic element. The multipole expansion of the magnetic field can be written in complex form as $\left(B_{y}+\mathrm{i} B_{x}\right)=C_{n-1}(x+\mathrm{i} y)^{n-1}$ and for $n=4$ we retrieve the octupole field. An electron, traveling in positive $z$-direction will get a transverse deflection according to
TABLE II. Assumed parameters and errors.

\begin{tabular}{lc}
\hline \hline Parameter [unit] & value \\
\hline BPM resolution [nm] & 50 \\
Active length of accelerating structure, $l[\mathrm{~m}]$ & 0.23 \\
Integrated octupole strength, $C_{3} l\left[\mathrm{kTm} / \mathrm{m}^{3}\right]$ & 73.5 \\
Distance to BPM, $L[\mathrm{~m}]$ & 3 \\
Half-distance between the centers of two & \\
accelerating structures, $\Delta L[\mathrm{~m}]$ & 0.125 \\
Error in position shift, $\sigma_{\Delta \hat{X}}, \sigma_{\Delta \hat{Y}}[\mathrm{~nm}]$ & 71 \\
Error in $K L, \sigma_{K L}[\mathrm{rel}]$. & $0.1 \%$ \\
\hline \hline
\end{tabular}

$$
\Delta x^{\prime}-\mathrm{i} \Delta y^{\prime}=K(x+\mathrm{i} y)^{3}
$$

where $K=\frac{C_{3} l}{(B \rho)}$ is the integrated octupole strength normalized to beam energy and we have assumed a thin lens approximation. This can be justified by numerical integrations that show that the relative error due to higher order effects is on the order of $2 \times 10^{-4}$ with the parameters used. The active length of the CLIC accelerating structure is $0.23 \mathrm{~m}$ and the integrated octupole strength normalized to beam energy is $K=2450 \mathrm{~m}^{-3}$, see Table II.

At a distance $L$ downstream from the octupole field the particle will have shifted horizontal position $\Delta \hat{x}$ according to $\Delta \hat{x}=L \Delta x^{\prime}$ and similarly for vertical position shift $\Delta \hat{y}$. This position shift is the difference of the beam position with $\mathrm{rf}$ in the accelerating structure turned on and off. We determine the position shifts of the centroid of the beam $\Delta \hat{X}$ and $\Delta \hat{Y}$ by averaging over the particle distribution (denoted by angle brackets) in the accelerating structure

$$
\Delta \hat{X}-\mathrm{i} \Delta \hat{Y}=K L\left\langle(x+\mathrm{i} y)^{3}\right\rangle .
$$

We can expand these terms involving $x$ and $y$ and if we assume a Gaussian beam distribution we can easily evaluate the expectation values, e.g., by using the technique described in Appendix A in [13]. In particular we note that

$$
\begin{aligned}
\left\langle x^{3}\right\rangle & =X^{3}+3 X \sigma_{x}^{2} \\
\left\langle x y^{2}\right\rangle & =X Y^{2}+2 Y \sigma_{x y}+X \sigma_{y}^{2} \\
\left\langle x^{2}\right\rangle & =X^{2}+\sigma_{x}^{2} \\
\langle x y\rangle & =X Y+\sigma_{x y}
\end{aligned}
$$

where capital $X$ and $Y$ denote the first moments, i.e., $X=$ $\langle x\rangle$ etc. Then we obtain for the position change $\Delta \hat{X}$ and $\Delta \hat{Y}$ at a distance $L$ downstream the octupole field as a function of the beam centroid position in accelerating structure

$$
\Delta \hat{X}-\mathrm{i} \Delta \hat{Y}=K L\left[(X+\mathrm{i} Y)^{3}+3\left(\sigma_{x}^{2}-\sigma_{y}^{2}+2 \mathrm{i} \sigma_{x y}\right)(X+\mathrm{i} Y)\right] .
$$

We note that the position shifts of the centroid of the beam at a distance $L$ from the octupole field is dependent on both 
the beam centroid transverse position $X$ and $Y$ and the beam size at the location of the octupole.

\section{FINDING THE CENTER OF A SINGLE STRUCTURE}

In the previous section we saw that the beam deflection due to the octupole field depends on the transverse position inside the accelerating structure. By moving the girder which the accelerating structures are mounted on, or by moving the beam parallel to the beam axis using two steering magnets or movable quadrupoles, we can control the transverse beam position inside the octupole $(X, Y)$. The geometry of the setup is shown in Fig. 1. We then measure the beam position shifts $\Delta \hat{X}$ and $\Delta \hat{Y}$ using a beam position monitor (BPM) downstream of the structure. From now on we will assume that BPMs are used for measuring the beam centroid position but screens or wire scanners also work. In the following we assume that initially the beam follows a trajectory that was determined by external means, for example by aligning to the center of the quadrupoles. We then seek to determine the horizontal offset $\tilde{X}$ and vertical offset $\tilde{Y}$ with respect to this trajectory. Including the offsets we write (2) as

$$
\Delta \hat{X}-\mathrm{i} \Delta \hat{Y}=K L\left\langle[(x-\tilde{X})+\mathrm{i}(y-\tilde{Y})]^{3}\right\rangle .
$$

The right-hand side of (5) depends on five unknown variables: the horizontal and vertical offsets $(\tilde{X}, \tilde{Y})$ and, like (4), on the beam size $\sigma_{x}^{2}, \sigma_{y}^{2}$ and correlation $\sigma_{x y}$. If we expand, calculate all expectation values and collect known and measurable parameters on the left-hand side, we obtain

$$
\begin{aligned}
\Delta \hat{X}- & \mathrm{i} \Delta \hat{Y}-K L(X+\mathrm{i} Y)^{3} \\
= & K L\left\{3(\tilde{X}+\mathrm{i} \tilde{Y})\left(\sigma_{y}^{2}-\sigma_{x}^{2}-2 \mathrm{i} \sigma_{x y}\right)-(\tilde{X}+\mathrm{i} \tilde{Y})^{3}\right. \\
& +3\left[(\tilde{X}+\mathrm{i} \tilde{Y})^{2}-\left(\sigma_{y}^{2}-\sigma_{x}^{2}-2 \mathrm{i} \sigma_{x y}\right)\right](X+\mathrm{i} Y) \\
& \left.-3(\tilde{X}+\mathrm{i} \tilde{Y})(X+\mathrm{i} Y)^{2}\right\} .
\end{aligned}
$$

The expression in (6) can be cast in the form of a linear least squares fit

$$
z=k_{1}+k_{2}(X+\mathrm{i} Y)+k_{3}(X+\mathrm{i} Y)^{2}
$$

with the unknown quantities $k_{1}, k_{2}, k_{3}$ given by

$$
\begin{aligned}
& k_{1}=K L\left[3(\tilde{X}+\mathrm{i} \tilde{Y})\left(\sigma_{y}^{2}-\sigma_{x}^{2}-2 \mathrm{i} \sigma_{x y}\right)-(\tilde{X}+\mathrm{i} \tilde{Y})^{3}\right] \\
& k_{2}=3 K L\left[(\tilde{X}+\mathrm{i} \tilde{Y})^{2}-\left(\sigma_{y}^{2}-\sigma_{x}^{2}-2 \mathrm{i} \sigma_{x y}\right)\right] \\
& k_{3}=-3 K L(\tilde{X}+\mathrm{i} \tilde{Y})
\end{aligned}
$$

on the right-hand side. Note that $k_{3}$ directly contains the sought offsets $\tilde{X}$ and $\tilde{Y}$. Furthermore, additional kicks from, e.g., quadrupole or dipole have an effect on $k_{1}$ and $k_{2}$ but leave $k_{3}$ unaffected. Note also that the beam sizes do not enter in $k_{3}$ which carries the information of the misalignment and the increased emittance when running at low energy will not affect it. The left-hand side contains the measured position shifts $\Delta \hat{X}$ and $\Delta \hat{Y}$ as well as the beam positions $X$ and $Y$ that we control with the steering magnets. We have

$$
z=\Delta \hat{X}-\mathrm{i} \Delta \hat{Y}-K L(X+\mathrm{i} Y)^{3} .
$$

In order to determine $\vec{k}=\left(k_{1}, k_{2}, k_{3}\right)$ we scan the beam transversely, i.e. make a series of measurements with different transverse positions $\left(X_{i}, Y_{i}\right)$. Hence we can express the fit as $\vec{z}=A \vec{k}$ where $\vec{z}$ is a column vector containing the measured position shifts, i.e. $z_{i}=\Delta \hat{X}_{i}-$ $\mathrm{i} \Delta \hat{Y}_{i}-K L\left(X_{i}+\mathrm{i} Y_{i}\right)^{3}$ and $A$ is a matrix with the monomials evaluated at each scan step, i.e., horizontal and vertical beam centroid position of the beam in the structure. If we perform a position scan of $N$ steps we obtain

$$
\left[\begin{array}{c}
z_{1} \\
z_{2} \\
\vdots \\
\vdots \\
z_{N}
\end{array}\right]=\left[\begin{array}{ccc}
1 & \left(X_{1}+\mathrm{i} Y_{1}\right) & \left(X_{1}+\mathrm{i} Y_{1}\right)^{2} \\
1 & \left(X_{2}+\mathrm{i} Y_{2}\right) & \left(X_{2}+\mathrm{i} Y_{2}\right)^{2} \\
\vdots & \vdots & \vdots \\
\vdots & \vdots & \vdots \\
1 & \left(X_{N}+\mathrm{i} Y_{N}\right) & \left(X_{N}+\mathrm{i} Y_{N}\right)^{2}
\end{array}\right]\left[\begin{array}{c}
k_{1} \\
k_{2} \\
k_{3}
\end{array}\right] .
$$

We can find the fit parameters $k_{i}$ of the least squares solution using the pseudo inverse and write the solution as $\vec{k}=\left(A^{\dagger} A\right)^{-1} A^{\dagger} \vec{z}$, where the dagger denotes the Hermitian

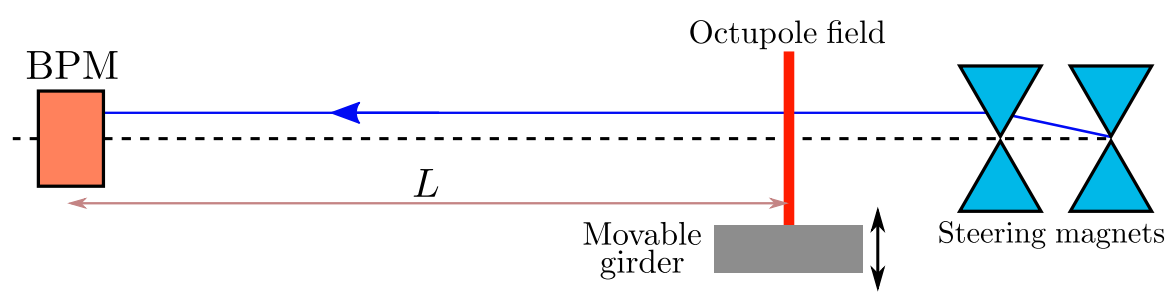

FIG. 1. Setup with a single accelerating structure with an octupole field. The beam travels from right to left and can be scanned transversely and parallel to the beam axis by moving the girder or by using two upstream steering magnets. The beam position shifts can be measured at a distance $L$ downstream from the octupole with a beam position monitor (BPM). 
transpose. The offsets $\tilde{X}$ and $\tilde{Y}$ can be extracted from the real and imaginary parts of fit parameter $k_{3}$ as indicated in the last equation of (8). To what accuracy this can be achieved is the subject of the next section.

\section{TOLERANCES}

In order to make the error analysis more tractable we formulate the fit problem in (10) to address real and imaginary components individually. Writing the vector $\vec{z}$ as $\vec{z}=\operatorname{Re}(\vec{z})+\mathrm{i} \operatorname{Im}(\vec{z})$ and splitting both the matrix $A=$ $B+\mathrm{i} C$ and $\vec{k}=\operatorname{Re}(\vec{k})+\mathrm{i} \operatorname{Im}(\vec{k})$ we rewrite (10) as

$$
\begin{aligned}
\operatorname{Re}(\vec{z})+\mathrm{i} \operatorname{Im}(\vec{z}) & =(B+\mathrm{i} C)(\operatorname{Re}(\vec{k})+\mathrm{i} \operatorname{Im}(\vec{k})) \\
& =B \operatorname{Re}(\vec{k})-C \operatorname{Im}(\vec{k})+\mathrm{i}(B \operatorname{Im}(\vec{k})+C \operatorname{Re}(\vec{k}))
\end{aligned}
$$

or, in component form

$$
\left[\begin{array}{c}
\operatorname{Re}\left(z_{1}\right) \\
\vdots \\
\operatorname{Re}\left(z_{N}\right) \\
\operatorname{Im}\left(z_{1}\right) \\
\vdots \\
\operatorname{Im}\left(z_{N}\right)
\end{array}\right]=\left[\begin{array}{cc}
B & -C \\
C & B
\end{array}\right]\left[\begin{array}{c}
\operatorname{Re}\left(k_{1}\right) \\
\operatorname{Re}\left(k_{2}\right) \\
\operatorname{Re}\left(k_{3}\right) \\
\operatorname{Im}\left(k_{1}\right) \\
\operatorname{Im}\left(k_{2}\right) \\
\operatorname{Im}\left(k_{3}\right)
\end{array}\right]
$$

where $N$ is the number of individual measurements. We denote the vector on the left-hand side by $\vec{Z}$ and the matrix and vector on the right-hand side by $D$ and $\vec{K}$, respectively.

In order to calculate the error bars for the fit parameters $\vec{K}$ we need to determine the error bars of the quantities $\vec{Z}$ on the left-hand side of (12), which in turn depend on the measured beam positions $\Delta \hat{X}$ and $\Delta \hat{Y}$, the excitation $K L$ and the positions in the structure $X, Y$ through (9). Collectively we denote these parameters by $\vec{x}=$ $(\Delta \hat{X}, \Delta \hat{Y}, X, Y, K L)$. Separating real and imaginary part we find

$\vec{Z}=\left[\begin{array}{c}\operatorname{Re}\left(z_{1}\right) \\ \vdots \\ \operatorname{Re}\left(z_{N}\right) \\ \operatorname{Im}\left(z_{1}\right) \\ \vdots \\ \operatorname{Im}\left(z_{N}\right)\end{array}\right]=\left[\begin{array}{c}\Delta \hat{X}_{1}-K L X_{1}^{3}+3 K L X_{1} Y_{1}^{2} \\ \vdots \\ \Delta \hat{X}_{N}-K L X_{N}^{3}+3 K L X_{N} Y_{N}^{2} \\ -\Delta \hat{Y}_{1}+K L Y_{1}^{3}-3 K L X_{1}^{2} Y_{1} \\ \vdots \\ -\Delta \hat{Y}_{N}+K L Y_{N}^{3}-3 K L X_{N}^{2} Y_{N}\end{array}\right]$

If we make the reasonable assumption that the errors of the parameters $\vec{x}$ are uncorrelated and given by the rms values in Table II we can express the covariance matrix $C_{x x}$ as a diagonal matrix containing the squares of the rms error bars of the $\vec{x}$ on the diagonal. We define $\vec{\sigma}_{\vec{x}}=$ $\left(\sigma_{\Delta \hat{X}}^{2}, \sigma_{\Delta \hat{Y}}^{2}, \sigma_{X}^{2}, \sigma_{Y}^{2}, \sigma_{K L}^{2}\right)$ and obtain

$$
C_{x x}=\operatorname{diag}\left(\vec{\sigma}_{\vec{x}}, \ldots, \vec{\sigma}_{\vec{x}}\right)
$$

where $C_{x x}$ is a $5 N \times 5 N$ matrix. In order to find the covariance matrix for $\vec{Z}$ we need to propagate $C_{x x}$ with the Jacobi matrix that relates the change in variables $\vec{x}$ to that in $\vec{Z}$ for which we need the partial derivatives

$$
\begin{aligned}
& \left.j_{\mathrm{R}}\left(\vec{x}_{i}\right) \equiv \frac{\partial \operatorname{Re}\left(z_{i}\right)}{\partial \vec{x}}\right|_{\vec{x}_{i}} \\
& =\left[\begin{array}{llll}
1 & 0 & 3 K L\left(Y_{i}^{2}-X_{i}^{2}\right) & 6 K L X_{i} Y_{i}-X_{i}^{3}+3 X_{i} Y_{i}^{2}
\end{array}\right] \\
& \left.j_{\mathrm{I}}\left(\vec{x}_{i}\right) \equiv \frac{\partial \operatorname{Im}\left(z_{i}\right)}{\partial \vec{x}}\right|_{\vec{x}_{i}} \\
& =\left[\begin{array}{lllll}
0 & -1 & -6 K L X_{i} Y_{i} & 3 K L\left(Y_{i}^{2}-X_{i}^{2}\right) & Y_{i}^{3}-3 X_{i}^{2} Y_{i}
\end{array}\right]
\end{aligned}
$$

where the elements containing KL are small compared to 1 and -1 . Therefore the errors are dominated by the errors in $\Delta \hat{X}$ and $\Delta \hat{Y}$. Then $j_{\mathrm{R}}\left(\vec{x}_{i}\right)$ and $j_{\mathrm{I}}\left(\vec{x}_{i}\right)$ populate the Jacobi matrix according to

$$
J=\left[\begin{array}{cccc}
\vec{j}_{\mathrm{R}}\left(\vec{x}_{1}\right) & 0 & \cdots & 0 \\
0 & \vec{j}_{\mathrm{R}}\left(\vec{x}_{2}\right) & & \vdots \\
\vdots & & \ddots & 0 \\
0 & \cdots & 0 & \vec{j}_{\mathrm{R}}\left(\vec{x}_{N}\right) \\
\vec{j}_{\mathrm{I}}\left(\vec{x}_{1}\right) & 0 & \cdots & 0 \\
0 & \vec{j}_{\mathrm{I}}\left(\vec{x}_{2}\right) & & \vdots \\
\vdots & & \ddots & 0 \\
0 & \cdots & 0 & \vec{j}_{\mathrm{I}}\left(\vec{x}_{N}\right)
\end{array}\right]
$$

The covariance matrices propagate in a similar way as the beam matrices, but with the Jacobi matrix instead of the transfer matrix [19]. Therefore, the covariance matrix of $\vec{Z}$, which contains all correlations among the measurements consistently, is given by

$$
C_{Z Z}=J C_{x x} J^{T} .
$$

Finally we determine the fit parameters $\vec{K}$ according to [20] 


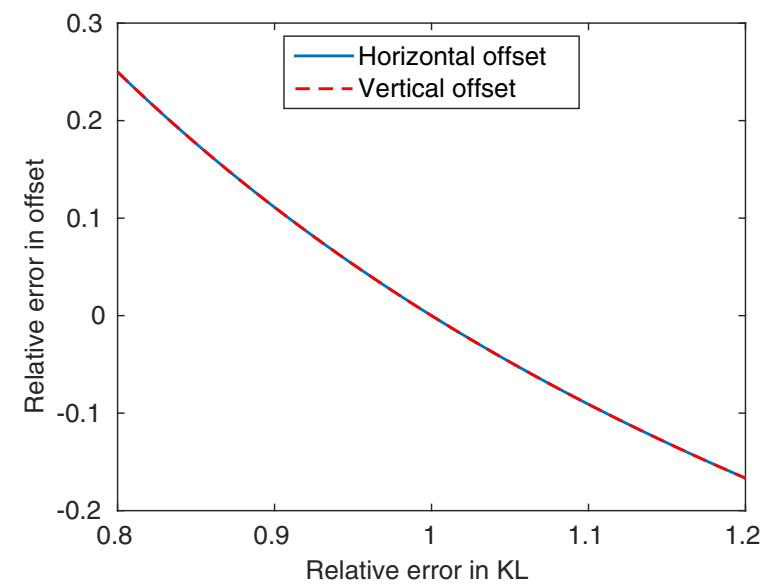

FIG. 2. Relative error in fitted offset for different systematic errors in $K L$.

$$
\vec{K}=\left(D^{T} C_{Z Z}^{-1} D\right)^{-1} D^{T} C_{Z Z}^{-1} \vec{Z}
$$

The errors in $\vec{K}$ are given by the diagonal elements of the covariance matrix of $K$ which leads us to [20]

$$
C_{K K}=\left(D^{T} C_{Z Z}^{-1} D\right)^{-1}
$$

From (8) we relate the error bar of the fit parameters $\vec{k}$ to those of the sought alignment tolerances. We determine the error bar $\sigma_{\tilde{X}}$ of $\tilde{X}$ and $\sigma_{\tilde{Y}}$ of $\tilde{Y}$ to be

$$
\sigma_{\tilde{X}}=\frac{\sigma_{\operatorname{Re}\left(k_{3}\right)}}{3 K L} \quad \text { and } \quad \sigma_{\tilde{Y}}=\frac{\sigma_{\operatorname{Im}\left(k_{3}\right)}}{3 K L} .
$$

In order to estimate the achievable error of this method we insert numbers relevant for CLIC. We use the beam parameters of the CLIC main beam at the beginning of the main linac, listed in Table I. In Table II we show the assumed relative uncertainties. The uncertainty in $K L$ is dominated by the uncertainty in the integrated octupole strength $C_{3} l$ which in turn comes from rf jitter.

The CLIC accelerating structures have a minimum iris diameter of roughly $4 \mathrm{~mm}$ and we assume that the beam can be moved transversely $\pm 1 \mathrm{~mm}$ inside the accelerating structure. We point out that the horizontal $\mathrm{rms}$ beam size is $18 \mu \mathrm{m}$ and vertical rms beam size is $4 \mu \mathrm{m}$ only, as derived from values in Table I.
We assume a parallel scan procedure of 40 steps, we consider a scan path in the shape of a cross, i.e., first we scan 20 steps horizontally while keeping the vertical position fixed and then 20 steps vertically while keeping the horizontal position fixed. Then we follow the procedure described in (14)-(17) to determine the errors in $\vec{Z}$, i.e., the right-hand side of (13). It turns out that the error bar in each scan point is the same throughout the scan. This is not surprising since most terms in (15) are small. The leading elements are 1 and -1 , which means that the errors in $\Delta \hat{X}$ and $\Delta \hat{Y}$ will dominate the error in $\vec{Z}$. This also implies that we are the most sensitive to the BPM resolution and less so to random errors in $K L$ and position $X, Y$ in the structure.

Once we have the errors in $\vec{Z}$ we can use (19)-(20) to find the errors in the fitted offsets. We found them to be $1 \mu \mathrm{m}$ for both the horizontal and vertical offset. These results given by the analytical expressions were verified with a Monte Carlo analysis [18]. We can see that this result compares favorably with the tolerance for beam alignment in the accelerating structures for CLIC which is $5 \mu \mathrm{m}$ in order to ensure high luminosity [1]. We also considered the effect of errors in $X$ and $Y$ in the fit matrix $A$, i.e., right-hand side of (10), due to uncertainty in the beam position inside the accelerating structure. However, a Monte Carlo analysis with random noise with rms equal to BPM resolution added showed that this effect is negligible.

So far we have only investigated the effect on the alignment resolution due to random errors. Of course we are also sensitive to systematic errors in $K L$. The parameter $K L$ depends on the integrated octupole strength, which in turn depends on the rf power fed into the structure. This means rf power measurements and model calibration might induce systematic errors. We did a numerical analysis and found that there is an almost linear dependence on the error in $K L$ and the error in fitted offsets [18], see Fig. 2.

\section{TWO CONSECUTIVE OCTUPOLES}

In the previous section we showed how to determine the offsets for a single structure. However, in CLIC the accelerating structures are mounted together two and two and receive rf power from a single source [1]. Here we show how to disentangle the offsets of two accelerating structures simultaneously with the geometry shown in Fig. 3. If the distance between the two elements is small

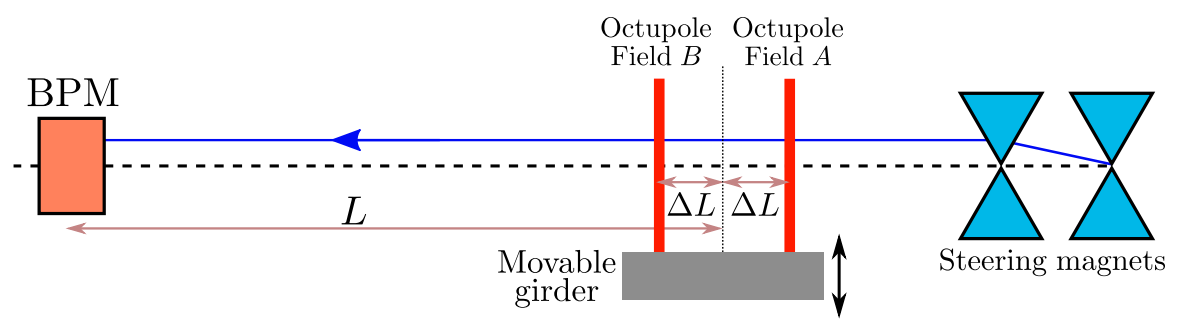

FIG. 3. Aligning the beam in two accelerating structures both with octupole fields. 
we can ignore higher order terms and consider only the sum effect of two independent octupole kicks. In other words, if the second octupole is close to the first, the kick from the second octupole is only weakly affected by the kick from the first.

In order to justify the approximation we calculate the additional kick due to the small horizontal position shift $\delta x$ in the second octupole field which is given by

$$
\delta x=-2 K \Delta L \tilde{X}_{A}^{3}
$$

where $2 \Delta L$ is the distance between the two structures and we assume that the incoming beam is on axis and the kick is due to the misalignment $\tilde{X}_{A}$. The integrated octupole strengths of the two structures are assumed to be the same since they receive rf power from the same source, we denote $K_{A}=K_{B}=K$. Then the kick from both octupole fields becomes

$$
\begin{aligned}
\Delta x^{\prime} & =K\left(-\tilde{X}_{A}\right)^{3}+K\left(-2 K \Delta L \tilde{X}_{A}^{3}-\tilde{X}_{B}\right)^{3} \\
& \approx-K\left(\tilde{X}_{A}^{3}+\tilde{X}_{B}^{3}\right)-6 K^{2} \Delta L \tilde{X}_{B}^{2} \tilde{X}_{A}^{3}
\end{aligned}
$$

where the first term corresponds to the approximation we will use and the second term describes the perturbation in the angle due to the small position shift in the second octupole. On a downstream BPM we see beam position to move by this angle leveraged by the distance $L$. In order to estimate the magnitude of the respective terms we assume $\tilde{X}_{A}=\tilde{X}_{B}=\tilde{X}$ and calculate the ratio $r$ of the first and second order with the result

$$
r=3 K \Delta L \tilde{X}^{2} .
$$

We use $\Delta L=0.125 \mathrm{~m}$ and a large offset $\tilde{X}=0.5 \mathrm{~mm}$ to be conservative, which together with values from the previous section gives $r$ to be less than $3 \times 10^{-4}$.

From (6) we can then write a general expression for position shifts due to two independent, consecutive octupole kicks as

$$
\begin{aligned}
\Delta \hat{X}- & \mathrm{i} \Delta \hat{Y}-K(L+\Delta L)\left(X_{A}+\mathrm{i} Y_{A}\right)^{3} \\
- & K(L-\Delta L)\left(X_{B}+\mathrm{i} Y_{B}\right)^{3} \\
= & K(L+\Delta L)\left\{3\left(\tilde{X}_{A}+\mathrm{i} \tilde{Y}_{A}\right) c-\left(\tilde{X}_{A}+\mathrm{i} \tilde{Y}_{A}\right)^{3}\right. \\
& +3\left[\left(\tilde{X}_{A}+\mathrm{i} \tilde{Y}_{A}\right)^{2}-c\right]\left(X_{A}+\mathrm{i} Y_{A}\right) \\
& \left.-3\left(\tilde{X}_{A}+\mathrm{i} \tilde{Y}_{A}\right)\left(X_{A}+\mathrm{i} Y_{A}\right)^{2}\right\} \\
& +K(L-\Delta L)\left\{3\left(\tilde{X}_{B}+\mathrm{i} \tilde{Y}_{B}\right) c-\left(\tilde{X}_{B}+\mathrm{i} \tilde{Y}_{B}\right)^{3}\right. \\
& +3\left[\left(\tilde{X}_{B}+\mathrm{i} \tilde{Y}_{B}\right)^{2}-c\right]\left(X_{B}+\mathrm{i} Y_{B}\right) \\
& \left.-3\left(\tilde{X}_{B}+\mathrm{i} \tilde{Y}_{B}\right)\left(X_{B}+\mathrm{i} Y_{B}\right)^{2}\right\}
\end{aligned}
$$

where we have used subscript $A$ for the first accelerating structure and subscript $B$ for the second. We introduce $c=\sigma_{y}^{2}-\sigma_{x}^{2}-2 \mathrm{i} \sigma_{x y}$, which for now we assume to be known and to be the same in the two octupoles which is reasonable as long as $\Delta L / \beta$ is small where $\beta$ is the beta function at the position of the accelerating structure. The transverse position inside the first accelerating structure is denoted $\left(X_{A}, Y_{A}\right)$ and the offsets in the first accelerating structure is denoted $\left(\tilde{X}_{A}, \tilde{Y}_{A}\right)$. Similarly for the second structure with subscript $B$.

We deploy the same procedure as before and scan the beam in parallel to the beam axis, see Fig. 3. In that case we have that $X_{A}=X_{B}=X$ and $Y_{A}=Y_{B}=Y$. For a more compact notation we write the offsets in complex form $\tilde{Z}_{A}=\tilde{X}_{A}+\mathrm{i} \tilde{Y}_{A}$ and $\tilde{Z}_{B}=\tilde{X}_{B}+\mathrm{i} \tilde{Y}_{B}$, then (24) becomes

$$
\begin{aligned}
\Delta \hat{X}- & \mathrm{i} \Delta \hat{Y}-2 K L(X+\mathrm{i} Y)^{3} \\
= & K L\left\{3\left(1+\frac{\Delta L}{L}\right) \tilde{Z}_{A} c+3\left(1-\frac{\Delta L}{L}\right) \tilde{Z}_{B} c\right. \\
& -\left(1+\frac{\Delta L}{L}\right) \tilde{Z}_{A}^{3}-\left(1-\frac{\Delta L}{L}\right) \tilde{Z}_{B}^{3} \\
& +3\left[\left(1+\frac{\Delta L}{L}\right) \tilde{Z}_{A}^{2}+\left(1-\frac{\Delta L}{L}\right) \tilde{Z}_{B}^{2}-2 c\right](X+\mathrm{i} Y) \\
& \left.-3\left[\left(1+\frac{\Delta L}{L}\right) \tilde{Z}_{A}+\left(1-\frac{\Delta L}{L}\right) \tilde{Z}_{B}\right](X+\mathrm{i} Y)^{2}\right\}
\end{aligned}
$$

which again is a fit in the form of (7). From fit parameters $k_{2}$ and $k_{3}$ we get

$$
\begin{aligned}
& k_{2}=3 K L\left[\left(1+\frac{\Delta L}{L}\right) \tilde{Z}_{A}^{2}+\left(1-\frac{\Delta L}{L}\right) \tilde{Z}_{B}^{2}-2 c\right] \\
& k_{3}=-3 K L\left[\left(1+\frac{\Delta L}{L}\right) \tilde{Z}_{A}+\left(1-\frac{\Delta L}{L}\right) \tilde{Z}_{B}\right] .
\end{aligned}
$$

We denote the sum and difference between the two offsets as $\tilde{Z}=\tilde{Z}_{A}+\tilde{Z}_{B}$ and $\Delta \tilde{Z}=\tilde{Z}_{B}-\tilde{Z}_{A}$. Rewriting (26) we obtain

$$
\begin{aligned}
\tilde{Z}^{2}+(\Delta \tilde{Z})^{2}-\frac{2 \Delta L}{L} \tilde{Z} \Delta \tilde{Z} & =\frac{2 k_{2}}{3 K L}+4 c \\
\tilde{Z}-\frac{\Delta L}{L} \Delta \tilde{Z} & =\frac{-k_{3}}{3 K L}
\end{aligned}
$$

and inserting the second equation into the first gives

$\Delta \tilde{Z}= \pm\left\{\left[1-\left(\frac{\Delta L}{L}\right)^{2}\right]\left[\frac{2 k_{2}}{3 K L}-\left(\frac{k_{3}}{3 K L}\right)^{2}-2 c\right]\right\}^{\frac{1}{2}}$

which shows that we can determine the magnitude of the difference of the offsets but not the sign. We define $\Delta \tilde{Z}^{\prime}$ to denote the magnitude of the difference of the offsets. Then from (27)-(28) we conclude 


$$
\begin{aligned}
\tilde{Z} & =\frac{-k_{3}}{3 K L} \pm \frac{\Delta L}{L} \Delta \tilde{Z}^{\prime} \\
\Delta \tilde{Z}^{\prime} & =\left\{\left[1-\left(\frac{\Delta L}{L}\right)^{2}\right]^{-1}\left[\frac{2 k_{2}}{3 K L}-\left(\frac{k_{3}}{3 K L}\right)^{2}+4 c\right]\right\}^{\frac{1}{2}} .
\end{aligned}
$$

We have divided the misalignment into two contributions, global $\tilde{Z}$ and relative $\Delta \tilde{Z}^{\prime}$. The term $\Delta \tilde{Z}^{\prime}$ is the misalignment of the two structures relative to each other, i.e., a measure of the angle between the line through the centers of the two structures and the reference orbit. $\tilde{Z}$ is a measure of the distance between the line through the centers of the two structures and the reference orbit.

The procedure of alignment consists of first verifying the relative alignment of the structures by confirming that $\Delta \tilde{Z}^{\prime}$ is small. If $\Delta \tilde{Z}^{\prime}$ is large the relative alignment of the structures has to be improved by moving the support of the structures or by moving the individual structures. Once the relative alignment is ensured we can adjust the beam orbit to minimize the global offset $\tilde{Z}$. We note that if $\Delta \tilde{Z}^{\prime}=0$ we have $\tilde{Z}_{A}=\tilde{Z}_{B}=\tilde{Z} / 2$.

To determine the tolerance we follow the same procedure as before to determine the errors in the real and imaginary parts of the fit parameters $k_{2}$ and $k_{3}$. If we use the same scan procedure as in Sec. IV we get the same errors in the fit parameters. Then we compute the Jacobi matrix in order to propagate these errors to those for real and imaginary parts of $\tilde{Z}$ and $\Delta \tilde{Z}^{\prime}$. Since $\Delta \tilde{Z}^{\prime}$ contains a square root this means that the error will grow as $\Delta \tilde{Z}^{\prime}$ decreases. Thus there is a point when the error in the difference in offsets $\sigma_{\Delta \tilde{Z}^{\prime}}$ becomes as large as the difference in offsets itself $\Delta \tilde{Z}^{\prime}$. We find this to be $26 \mu \mathrm{m}$ and we interpret this to be our limit in precision for the relative alignment. We express the precision in terms of angle and we note that $26 \mu \mathrm{m}$ over the distance of $2 \Delta L$ corresponds to an angle of $0.1 \mathrm{mrad}$.

In $\tilde{Z}$ the term $\Delta \tilde{Z}^{\prime}$ is scaled with $\frac{\Delta L}{L}$ which reduces the error. We find the error in $\tilde{Z}$ to be $1.5 \mu \mathrm{m}$, to be compared to $1 \mu \mathrm{m}$ in the case of a single octupole. Thus we conclude that the relative alignment of the two structures can be determined with a tolerance on the order of $26 \mu \mathrm{m}$ and once this is done the global alignment can be determined with a tolerance on the order of $1.5 \mu \mathrm{m}$. All the analytical results of the error propagation were verified with a multiparticle Monte Carlo code. We note that the misalignment in this case depends on the beam sizes through the parameter $c$ and running at low energy with increased emittance will affect our method. To remedy this we can either measure the beam sizes on nearby screens, if those are available, or sacrifice the information about the internal misalignment $\Delta \tilde{Z}$ and only determine the average misalignment of the structure $\tilde{Z}$ by interpreting the detected kick as coming from single octupolar perturbation halfway between the structures.
Apart from scanning parallel to the beam axis we tried to scan the angle of the beam. With the two upstream steering magnets we change the angle of the incoming beam while keeping the position halfway in between the two structures fixed. This gave similar results as (29) but with $\tilde{Z}$ and $\Delta \tilde{Z}$ interchanged, thus in this case, only the magnitude of the sum of the offsets could be determined. Then one would do the opposite as in the case of a parallel scan; first minimize the global offset $\tilde{Z}$ and then determine the relative alignment $\Delta \tilde{Z}$ including the sign. However, aperture restrictions near the BPM (beam pipe radius of the CLIC main beam is $4 \mathrm{~mm}$ [1]) limit how much the beam angle can be changed resulting in small transverse displacements of the beam inside the structures. This leads to considerably larger errors in the fit parameters $k_{2}$ and $k_{3}$ compared to a parallel scan. Even worse so, $\Delta \tilde{Z}$ scales unfavorably with $L / \Delta L$. All together this gave tolerances of a factor 100 worse compared to a parallel scan.

We also attempted a scan path that is a linear combination of parallel and angle scan. There is an optimum path where we can determine both $\tilde{Z}$ and $\Delta \tilde{Z}$ including their signs. But again, the errors increased compared to a parallel scan because of limited scan range due to aperture constraints. Summarizing, parallel scan is the best we can do since then we utilize maximum transverse displacement of the beam inside the acceleration structure. In another setup with less restrictions in beam displacement other scan paths might be worth to consider.

\section{CONCLUSIONS}

We have demonstrated a method to utilize the octupole component of the rf fields in accelerating structures for beam-based alignment. By moving the girders with the accelerating structures or by moving the beam with two steering magnets or quadrupole movers while switching the rf power on and off we can measure the beam position shifts for different transverse positions in the accelerating structure. This eliminates many systematic errors since we measure position shifts and not absolute positions, and furthermore since we can measure the shifts from consecutive shots without moving the beam. Another advantage of this method is that it requires no additional hardware which could be a big bonus considering the large number of accelerating structures in CLIC.

We propose to perform the alignment campaign with only one drive beam sector powered at a time thus ensuring that the alignment is done at moderate beam energy. Then the kick, which scales inversely with beam energy, has a sufficient magnitude to maintain accuracy of the procedure. We expect that balancing stability requirements for running the linac at low energy with requirements for sufficient resolution in later sectors, will require careful alignment and optimization of the upstream sectors. Running a low energy will certainly increase the emittances and beam 
sizes, but our method controls for the beam size, which affects $k_{1}$ and $k_{2}$, but not $k_{3}$ which carries the information about the misalignment for a single octupole. For the twostructure setup we discussed methods to handle this in the previous section.

We showed the method for a single structure and two consecutive structures. For the case of a single structure it is straightforward to determine the offset from a least squares fit of the measured position shifts. We assume a scan path where the beam is moved in parallel to the beam axis with a transverse displacement of $\pm 1 \mathrm{~mm}$. From error propagation we determine the achievable tolerances and find them to be of the order of $1 \mu \mathrm{m}$ for the CLIC main beam parameters. In the case of two consecutive accelerating structures it is possible to determine the magnitude of the relative alignment but not the sign. The alignment procedure consists of first minimizing the relative misalignment and then the global misalignment. The achievable tolerances were found to be $1.5 \mu \mathrm{m}$ for the global misalignment and about 20 times worse for the relative misalignment.

As pointed out earlier, our method is complementary to alignment using wakefield monitors and may even serve as a backup in case of hardware failure of the latter. Our method may also be used to align other proposed X-band linacs that are based on CLIC technology and operate at lower energy. Examples are linear accelerators for freeelectron lasers, industrial, and medical applications [21].

\section{ACKNOWLEDGMENTS}

The authors thank Alexej Grudiev for insightful discussions and Andrea Latina for help on using PLACET. The authors would like to thank the Knut och Alice Wallenberg Foundation and the Swedish Research Council under No. 2011-6305 and No. 2014-6360 for funding.

[1] S. Stapnes, Report No. CERN-2012-005, Geneva, 2012.

[2] R. Ruber, V. Ziemann, T. Ekelöf, A. Palaia, W. Farabolini, and R. Corsini, The CTF3 two-beam test stand, Nucl. Instrum. Methods Phys. Res., Sect. A 729, 546 (2013).

[3] P. Eliasson and D. Schulte, Design of main linac emittance tuning bumps for the Compact Linear Collider and the International Linear Collider, Phys. Rev. ST Accel. Beams 11, 011002 (2008).

[4] K. Kubo et al., in Proceedings of the Particle Accelerator Conference, Dallas, TX, 1995 (IEEE, New York, 1995), p. 3112.
[5] R. Jones et al., in Proceedings of the 2003 Particle Accelerator Conference, Portland, OR (IEEE, New York, 2003), p. 2760.

[6] J. Seeman et al., in Proceedings of the 1991 Particle Accelerator Conference, San Francisco, CA, 1991 (IEEE, New York, 1991), p. 2949.

[7] M. Seidel and C. Adolphsen, Precision beam to structure alignment in linear accelerators, AIP Conf. Proc. 472, 713 (1999).

[8] A. Latina, J. Pfingstner, D. Schulte, E. Adli, F. J. Decker, and N. Lipkowitz, Experimental demonstration of a global dispersion-free steering correction at the new linac test facility at SLAC, Phys. Rev. ST Accel. Beams 17, 042803 (2014).

[9] J. Huopana, Report No. CLIC-Note-777, 2007.

[10] R. Lillest $\varnothing 1$ et al., in Proceedings of the 7th International Particle Accelerator Conference (IPAC16), Busan, South Korea (JACoW, Geneva, Switzerland, 2016), WEOBB02, p. 2099.

[11] W. Farabolini and A. Grudiev, at the CLIC RF Development Meeting, http://indico.cern.ch/event/245687/, 2013.

[12] W. Farabolini et al., in Proceedings of the 5th International Particle Accelerator Conference (IPAC14), Dresden, Germany (2014) (JACoW, Dresden, Germany, 2014), WEOCA02, p. 1880.

[13] J. Ögren, R. Ruber, V. Ziemann, and W. Farabolini, Measuring the full transverse beam matrix using a single octupole, Phys. Rev. ST Accel. Beams 18, 072801 (2015).

[14] P. Bambade and R. Ericsson, 1986 Linear Accelerator Conference, p. 475 http://accelconf.web.cern.ch/ AccelConf/186/papers/th3-16.pdf.

[15] V. Ziemann, Report No. PEP-II/AP 3-92, 1992.

[16] I. Syratchev et al., in Proceedings of the 3rd International Particle Accelerator Conference, New Orleans, LA, 2012 (IEEE, Piscataway, NJ, 2012), TUPPR019, p. 1852.

[17] D. Schulte et al., The tracking code PLACET, July 2013 http://svnweb.cern.ch/world/wsvn/clicsw/trunk/ placet-documentation/placet.pdf.

[18] J. Ögren and V. Ziemann, in Proceedings of the 7th International Particle Accelerator Conference (IPAC16), Busan, South Korea (JACoW, Geneva, Switzerland, 2016), MOPMR022, p. 280.

[19] V. Ziemann, in Proceedings of the 7th International Particle Accelerator Conference (IPAC16), Busan, South Korea (JACoW, Geneva, Switzerland, 2011), THPMB040, p. 3324.

[20] Data Analysis in High Energy Physics: A Practical Guide to Statistical Methods (1), edited by O. Behnke, K. Kröninger, and G. Schott (Wiley-VCH, New York, 2013).

[21] G. D' Auria, in Proceedings of the 26th Linear Accelerator Conference (LINAC2012), Tel-Aviv, Israel (2012) (JACoW, Tel-Aviv, Israel, 2012), WE1A03, p. 724. 